\title{
Totally Chemo- and Regioselective Cobalt(I) Mediated Formal Intermolecular Cyclotrimerization of Alkynes
}

\author{
Gaëlle Chouraqui, Marc Petit, Corinne Aubert*, Max Malacria*
}

Université Pierre et Marie Curie (Paris 6)

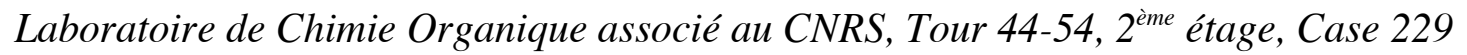

4, place de Jussieu 75252 Paris Cedex 05 - France

aubert@ccr.jussieu.fr ; malacria@ccr.jussieu.fr

\section{Supporting Information}


General Methods. Reactions were carried out under argon in flame-dried glassware, with magnetic stirring and degassed anhydrous solvents. All commercially available reagents were used without further purification unless otherwise noted. All solvents were reagent grade and distilled under positive pressure of dry nitrogen before use. THF was distilled from sodium/benzophenone. Solid reagents were dried in vacuo ( 0.5 to 0.1 $\mathrm{mmHg}$ ). - Thin layer chromatography (TLC) was performed on Merck $60 \mathrm{~F}_{254}$ silica gel. Merck Geduran SI $60 \AA$ Ailica gel (35-70 $\square \mathrm{m})$ was used for column chromatography according to Still's method. ${ }^{1} \mathrm{PE}$ and $\mathrm{EE}$ refer to petroleum ether and $\mathrm{Et}_{2} \mathrm{O}$. Chemical shifts are given in ppm, referenced to the residual proton resonances of the solvents $(\square=$ 7.26 for $\mathrm{CDCl}_{3} ; \mathrm{Z}=7.16$ for $\mathrm{C}_{6} \mathrm{D}_{6}$ ). Coupling constants $(J)$ are given in Hertz $(\mathrm{Hz})$. The terms $\mathrm{m}, \mathrm{s}, \mathrm{d}, \mathrm{t}, \mathrm{q}$, quint refer to multiplet, singlet, doublet, triplet, quartet, quintet; br means that the signal is broad. - Elemental analysis were performed by the Service Régional de Microanalyse de l'Université Pierre et Marie Curie - Low resolution mass spectra (MS) and high resolution mass spectra (HRMS) were measured by Service de spectrométrie de masse de l'ICSN-CNRS, Gif-sur-Yvette. Infrared spectra (IR) were recorded on a Bruker Tensor 27 spectrometer. Absorbance frequencies are given at maximum of intensity in $\mathrm{cm}^{-1}$.

\section{General procedure for the cyclization of diisopropylsilylethers :}

Cyclopentadienyldicarbonylcobalt(I) ( 0.05 equiv) was added to a boiling solution of triyne ( 1 equiv) in xylenes $(c=0.05 \mathrm{M})$ degassed by three freeze-pump-thaw cycles and was irradiated (light from projector lamp ; ELW, 300W, 50\% of its power). The reaction was monitored by TLC and after completion, the reaction mixture was concentrated in vacuo. The crude oil was purified by flash chromatography.

Cycloadduct (2): 70\%

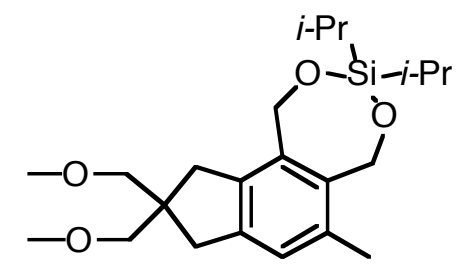

${ }^{1} \mathrm{H} \mathrm{NMR}\left(400 \mathrm{MHz}, \mathrm{CDCl}_{3}\right) \square 6.93$ (br s, 1H), 5.02 (s, 2H), 4.98 (s, 2H), 3.32 (br s, $16+4$ $\mathrm{H}), 2.82(\mathrm{~s}, 2 \mathrm{H}), 2.78(\mathrm{~s}, 2 \mathrm{H}), 2.34(\mathrm{~s}, 3 \mathrm{H}), 0.87-0.85(\mathrm{~m}, 14 \mathrm{H})$.

${ }^{13} \mathrm{C}$ NMR (100 MHz, $\mathrm{CDCl}_{3}$ ) $\mathrm{C} 142.1$ (2C), 138.9, 136.0, 134.7, 126.6, 76.0 (2C), 63.0, $61.3,59.3(2 \mathrm{C}), 47.8,38.9,37.3,19.8,16.8(4 \mathrm{C}), 13.0(2 \mathrm{C})$.

IR(neat) 3100, 2940, 1600, $1240 \mathrm{~cm}^{-1}$.

Anal. Calcd. for $\mathrm{C}_{22} \mathrm{H}_{36} \mathrm{O}_{4} \mathrm{Si}: \mathrm{C}, 67.30 ; \mathrm{H}, 9.24$. Found: C, 67.27; H, 9.02.

Cycloadduct (6a): $77 \%$.

${ }^{1}$ Still, W. C.; Khan, M.; Mitra, A. J. J. Org. Chem. 1978, 43, 2423-2425. 


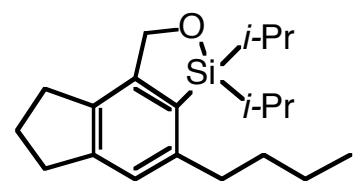

m. p. $43-45^{\circ} \mathrm{C}$.

${ }^{1} \mathrm{H}$ NMR (400 MHz, $\left.\mathrm{CDCl}_{3}\right) \square 7.07(\mathrm{~s}, 1 \mathrm{H}), 5.03(\mathrm{~s}, 2 \mathrm{H}), 2.94(\mathrm{t}, J=7.4 \mathrm{~Hz}, 2 \mathrm{H}), 2.73(\mathrm{t}$, $J=7.5 \mathrm{~Hz}, 2 \mathrm{H}), 2.63(\mathrm{t}, J=7.7 \mathrm{~Hz}, 2 \mathrm{H}), 2.15-2.11(\mathrm{~m}, 2 \mathrm{H}), 1.67-1.63(\mathrm{~m}, 2 \mathrm{H}), 1.47-1.43$ $(\mathrm{m}, 2 \mathrm{H}), 1.30-1.23(\mathrm{~m}, 2 \mathrm{H}), 1.08(\mathrm{~d}, J=7.3 \mathrm{~Hz}, 6 \mathrm{H}), 1.01-0.96(\mathrm{~d}, J=7.3 \mathrm{~Hz}, 6 \mathrm{H}$ overlaped with $\mathrm{t}, J=7.2 \mathrm{~Hz}, 3 \mathrm{H})$.

${ }^{13} \mathrm{C}$ NMR $\left(100 \mathrm{MHz}, \mathrm{CDCl}_{3}\right) \square 146.9,146.5,145.1,134.6,129.9,122.5,71.4,38.3,34.3$, $32.7,30.0,25.1,23.1,17.6(2 \mathrm{C}), 17.4(2 \mathrm{C}), 14.2,13.8(2 \mathrm{C})$.

IR (neat) 2926, 2861, 1461, 1066, 1042, 989, 880, 789, $661 \mathrm{~cm}^{-1}$.

Anal. Calcd. for $\mathrm{C}_{20} \mathrm{H}_{32} \mathrm{OSi}$ : C, 75.88; H, 10.19. Found: C, 75.60; H, 10.08 .

Cycloadduct (6b): $78 \%$.

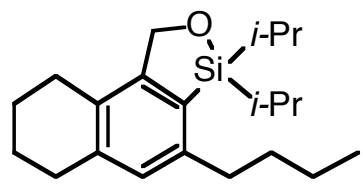

${ }^{1} \mathrm{H}$ NMR (400 MHz, $\left.\mathrm{CDCl}_{3}\right) \square 6.92(\mathrm{~s}, 1 \mathrm{H}), 5.02(\mathrm{~s}, 2 \mathrm{H}), 2.82(\mathrm{t}, J=5.8 \mathrm{~Hz}, 2 \mathrm{H}), 2.60(\mathrm{t}$, $J=8.3 \mathrm{~Hz}, 2 \mathrm{H}), 2.51(\mathrm{t}, J=5.4 \mathrm{~Hz}, 2 \mathrm{H}), 1.87-1.83(\mathrm{~m}, 4 \mathrm{H}), 1.68-1.64(\mathrm{~m}, 2 \mathrm{H}), 1.48-1.44$ $(\mathrm{m}, 2 \mathrm{H}), 1.29-1.25(\mathrm{~m}, 2 \mathrm{H}), 1.10(\mathrm{~d}, J=7.4 \mathrm{~Hz}, 6 \mathrm{H}), 1.01(\mathrm{~d}, J=7.4 \mathrm{~Hz}, 6 \mathrm{H}), 0.99(\mathrm{t}, J$ $=7.3 \mathrm{~Hz}, 3 \mathrm{H})$.

${ }^{13} \mathrm{C}$ NMR $\left(100 \mathrm{MHz}, \mathrm{CDCl}_{3}\right) \square 146.9,146.5,145.1,134.6,129.9,122.5,71.4,38.3$ 34.3, $32.7,30.0,25.1,23.1,23.0,17.6(2 \mathrm{C}), 17.4(2 \mathrm{C}), 14.2,13.8(2 \mathrm{C})$.

IR (neat) 2926, 2861, 1461, 1066, 1042, 989, 880, 789, $661 \mathrm{~cm}^{-1}$.

Anal. Calcd. for $\mathrm{C}_{21} \mathrm{H}_{34} \mathrm{OSi}$ : C, 76.30; H, 10.37. Found: C, 76.70; H, 9.88.

Cycloadduct (6c): $73 \%$.

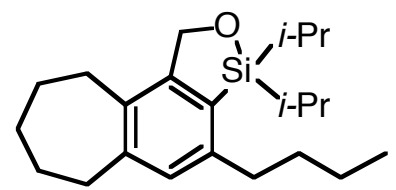

${ }^{1} \mathrm{H}$ NMR $\left(400 \mathrm{MHz}, \mathrm{CDCl}_{3}\right) \square 6.92(\mathrm{~s}, 1 \mathrm{H}), 5.13(\mathrm{~s}, 2 \mathrm{H}), 2.82(\mathrm{~m}, 2 \mathrm{H}), 2.62-2.56(\mathrm{~m}$, $4 \mathrm{H}), 1.88-1.83(\mathrm{~m}, 2 \mathrm{H}), 1.69-1.61(\mathrm{~m}, 6 \mathrm{H}), 1.44(\mathrm{~m}, 2 \mathrm{H}), 1.26(\mathrm{~m}, 2 \mathrm{H}), 1.09(\mathrm{~d}, J=7.3$ $\mathrm{Hz}, 6 \mathrm{H}), 1.01(\mathrm{~d}, J=7.3 \mathrm{~Hz}, 6 \mathrm{H}), 0.98(\mathrm{t}, J=7.3 \mathrm{~Hz}, 3 \mathrm{H})$.

${ }^{13} \mathrm{C}$ NMR $\left(100 \mathrm{MHz}, \mathrm{CDCl}_{3}\right) \square$ 148.0, 145.6, 144.0, 134.0, 128.9, 127.6, 72.0, 38.2 36.4, $34.0,32.7,29.7,28.0,27.4,23.0,17.5(2 \mathrm{C}), 17.3(2 \mathrm{C}), 14.1,13.8$ (2C).

IR (neat) 2923, 2861, 1462, 1061, 989, 879, 784, $675 \mathrm{~cm}^{-1}$.

Cycloadduct (6d): $67 \%$.

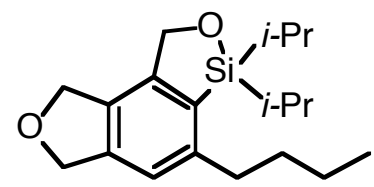


m.p. $71-73^{\circ} \mathrm{C}$

${ }^{1} \mathrm{H}$ NMR (400 MHz, $\left.\mathrm{CDCl}_{3}\right) \square 7.06(\mathrm{~s}, 1 \mathrm{H}), 5.13(\mathrm{~s}, 2 \mathrm{H}), 5.00(\mathrm{~s}, 4 \mathrm{H}), 2.67(\mathrm{t}, J=8.0 \mathrm{~Hz}$, $2 \mathrm{H}), 1.67-1.63(\mathrm{~m}, 2 \mathrm{H}), 1.47-1.41(\mathrm{~m}, 2 \mathrm{H}), 1.30-1.24(\mathrm{~m}, 2 \mathrm{H}), 1.08(\mathrm{~d}, J=7.3 \mathrm{~Hz}, 6 \mathrm{H})$, $0.99(\mathrm{~d}, J=7.5 \mathrm{~Hz}, 6 \mathrm{H}), 0.97(\mathrm{t}, J=7.3 \mathrm{~Hz}, 3 \mathrm{H})$.

${ }^{13} \mathrm{C}$ NMR $\left(100 \mathrm{MHz}, \mathrm{CDCl}_{3}\right) \square 146.7,143.9,142.0,131.1,129.9,118.9,73.6,72.0,70.8$, $38.2,34.1,23.0,17.5$ (2C), 17.3 (2C), 14.1, 13.7 (2C).

IR (neat) 2926, 2853, 1461, 1087, 900, 774, 687, $660 \mathrm{~cm}^{-1}$.

Cycloadduct (6e): $73 \%$.

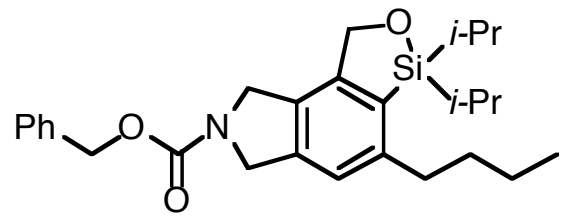

m.p. $71-73^{\circ} \mathrm{C}$

${ }^{1} \mathrm{H}$ NMR (400 MHz, $\left.\mathrm{CDCl}_{3}\right) \square 7.45-7.35(\mathrm{~m}, 5 \mathrm{H}), 7.11,7.05(2 \mathrm{~s}, 1 \mathrm{H}), 5.25(\mathrm{~s}, 2 \mathrm{H}), 5.04$, $5.01(2 \mathrm{~s}, 2 \mathrm{H}), 4.80,4.78(2 \mathrm{~s}, 2 \mathrm{H}), 4.64,4.60(2 \mathrm{~s}, 2 \mathrm{H}), 2.67(\mathrm{t}, J=8.0 \mathrm{~Hz}, 2 \mathrm{H}), 1.66-1.63$ $(\mathrm{m}, 2 \mathrm{H}), 1.48-1.43(\mathrm{~m}, 2 \mathrm{H}), 1.30-1.24(\mathrm{~m}, 2 \mathrm{H}), 1.09(\mathrm{~d}, J=7.2 \mathrm{~Hz}, 6 \mathrm{H}), 1.01-0.97(\mathrm{~m}$, $3 \mathrm{H}), 0.99(\mathrm{~d}, J=7.5 \mathrm{~Hz}, 6 \mathrm{H})$.

${ }^{13} \mathrm{C}$ NMR (100 MHz, $\left.\mathrm{CDCl}_{3}\right) \square 155.0,146.9,145.4,139.6,139.2,136.9,131.4,128.6$ (2C), 128.2 (2C), 128.0, 120.7, 70.7, 67.2, 52.6, 50.4, 38.3, 34.0, 23.0, 17.5 (2C), 17.3 (2C), 14.1, 13.7 (2C).

IR (neat) 3295, 2928, 2863, 1702, 1414, 1097, 1051, 780, 687, $661 \mathrm{~cm}^{-1}$.

Anal. Calcd. for $\mathrm{C}_{27} \mathrm{H}_{37} \mathrm{NO}_{3} \mathrm{Si}$ : C, 71.80; H, 8.26; N, 3.10. Found: C, 72.04; H, 8.31; N, 2.93

Cycloadduct (8): $65 \%$.

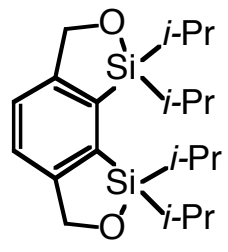

m.p. $71-73^{\circ} \mathrm{C}$

${ }^{1} \mathrm{H} \mathrm{NMR}\left(400 \mathrm{MHz}, \mathrm{CDCl}_{3}\right) \square 7.23(\mathrm{~s}, 2 \mathrm{H}), 5.18(\mathrm{~s}, 4 \mathrm{H}), 1.10(\mathrm{~d}, J=5.6 \mathrm{~Hz}, 14 \mathrm{H}), 0.92$ $(\mathrm{d}, J=6.9 \mathrm{~Hz}, 14 \mathrm{H})$.

${ }^{13} \mathrm{C}$ NMR (100 MHz, $\mathrm{CDCl}_{3}$ ) $\square 149.9$ (2C), 136.4 (2C), 132.4, 122.7, 72.5 (2C), 17.6 (8C), 14.3 (4C).

IR (neat) 2945, 2864, 1460, 1204, 1055, 781, 671, $620 \mathrm{~cm}^{-1}$.

Anal. Calcd. for $\mathrm{C}_{20} \mathrm{H}_{34} \mathrm{O}_{2} \mathrm{Si}_{2}$ : C, 66.24; H, 9.45. Found: C, 66.06; H, 9.29.

Cycloadduct (10): 77\%. 


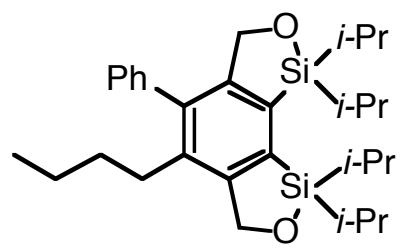

${ }^{1} \mathrm{H}$ NMR (400 MHz, $\left.\mathrm{CDCl}_{3}\right) \square 7.33-7.12(\mathrm{~m}, 5 \mathrm{H}), 5.04(\mathrm{~s}, 2 \mathrm{H}), 4.61(\mathrm{~s}, 2 \mathrm{H}), 2.38(\mathrm{~m}$, $2 \mathrm{H}), 1.15(\mathrm{~m}, 4 \mathrm{H}), 0.96(\mathrm{~m}, 28 \mathrm{H}), 0.57(\mathrm{t}, J=7.3 \mathrm{~Hz}, 3 \mathrm{H})$.

${ }^{13} \mathrm{C}$ NMR $\left(100 \mathrm{MHz}, \mathrm{CDCl}_{3}\right) \square 154.1,151.2,145.1,138.1,133.7,129.5,128.2$ (2C), 127.3 (2C), 126.0, 121.4, 72.9, 71.4, 35.4, 32.3, 22.0, 16.4 (2C), 16.3 (2C), 16.2 (2C), $16.0(2 \mathrm{C}), 12.8(2 \mathrm{C}), 12.5,12.2(2 \mathrm{C})$

IR (neat) 2937, 2862, 1461, 1062, 791, $637 \mathrm{~cm}^{-1}$.

Anal. Calcd. for $\mathrm{C}_{30} \mathrm{H}_{46} \mathrm{O}_{2} \mathrm{Si}_{2}$ : C, 72.81; H, 9.37. Found: C, 72.79; H, 9.37.

\section{Cycloadduct (12): 67\%.}

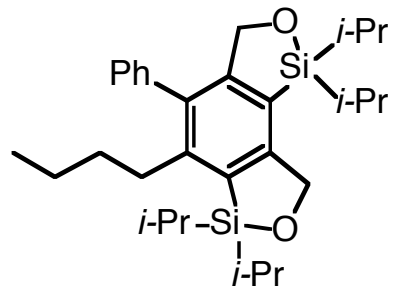

${ }^{1} \mathrm{H}$ NMR (400 MHz, $\left.\mathrm{CDCl}_{3}\right) \square 7.28(\mathrm{~m}, 3 \mathrm{H}), 7.13(\mathrm{~m}, 2 \mathrm{H}), 5.04(\mathrm{~s}, 2 \mathrm{H}), 4.61(\mathrm{~s}, 2 \mathrm{H})$, $2.38(\mathrm{~m}, 2 \mathrm{H}), 1.15(\mathrm{~m}, 4 \mathrm{H}), 0.95(\mathrm{~m}, 28 \mathrm{H}), 0.57(\mathrm{t}, J=14.6 \mathrm{~Hz}, 3 \mathrm{H})$.

${ }^{13} \mathrm{C}$ NMR $\left(100 \mathrm{MHz}, \mathrm{CDCl}_{3}\right) \square 153.1,151.4,145.1,138.1,133.7,129.5,128.2(2 \mathrm{C})$, 127.3(2C), 126.0, 121.4, 72.9, 71.4, 35.4, 32.3, 22.0, 16.4 (2C), 16.3 (2C), 16.2 (2C), 16.0 (2C), $12.8(2 \mathrm{C}), 12.5,12.2(2 \mathrm{C})$.

IR (neat) 2937, 2862, 1461, 1062, 791, $637 \mathrm{~cm}^{-1}$.

HRMS calcd for $\mathrm{C}_{30} \mathrm{H}_{46} \mathrm{O}_{2} \mathrm{Si}_{2}\left([\mathrm{M}+\mathrm{H}]^{+}\right)$calcd:495.3093. Found:495.3114. Calcd for ([M$\left.\mathrm{H}]^{+}\right)$: 493.2966. Found:493.2958.

Compound 13: 88\% over 2 steps from 3.

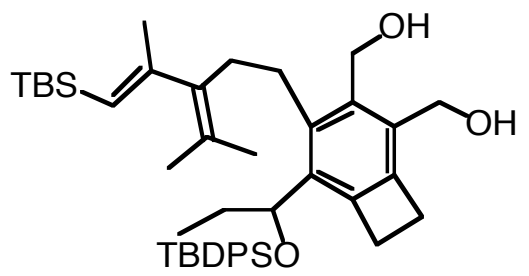

The cyclization of $\mathbf{3}$ was carried out following the procedure described above and furnished the cycloadduct $\mathbf{4}$ which was used without further purification.

To a cooled $\left(-78^{\circ} \mathrm{C}\right)$ solution of $\mathbf{4}$ in THF was added dropwise a $1 \mathrm{M}$ solution of TBAF (3 equiv). The solution was warmed up to $0^{\circ} \mathrm{C}$ and stirred until TLC had indicated the completion of the reaction. The reaction mixture was diluted with $\mathrm{Et}_{2} \mathrm{O}$, washed with a saturated solution of $\mathrm{NH}_{4} \mathrm{Cl}$, brine, dried over $\mathrm{MgSO}_{4}$, filtered and concentrated in vacuo. Flash chromatography $(\mathrm{PE} / \mathrm{AcOEt}=9 / 1)$ of the residue furnished the diol $\mathbf{1 3}$. 
${ }^{1} \mathrm{H}$ NMR (200 MHz, $\left.\mathrm{CDCl}_{3}\right) \square 7.69$ (d, $\left.J=6.8 \mathrm{~Hz}, 2 \mathrm{H}\right), 7.44-7.09$ (m, 8H), 5.09 (br s, $1 \mathrm{H}), 4.17$ (br s, 4H), $3.38(\mathrm{~m}, 3 \mathrm{H}), 3.15(\mathrm{~m}, 2 \mathrm{H}), 2.34(\mathrm{~m}, 2 \mathrm{H}), 2.00(\mathrm{~m}, 2 \mathrm{H}), 1.75$ (s, $3 \mathrm{H}), 1.62$ (s, 3H), $1.44(\mathrm{~s}, 3 \mathrm{H}), 1.07(\mathrm{~m}, 12 \mathrm{H}), 0.94(\mathrm{~m}, 11 \mathrm{H}), 0.13(\mathrm{~s}, 6 \mathrm{H})$.

IR(neat) 3329, 2927, 2857, 1742, 1599, 1055, 823, $700 \mathrm{~cm}^{-1}$.

HRMS calcd for $\mathrm{C}_{44} \mathrm{H}_{64} \mathrm{O}_{3} \mathrm{Si}_{2}\left(\left[\mathrm{M}+\mathrm{NH}_{4}^{+}\right]\right): 714.4738$. Found : 714.4755 .

\section{(6-Butyl-indan-4-yl)-methanol (14):}

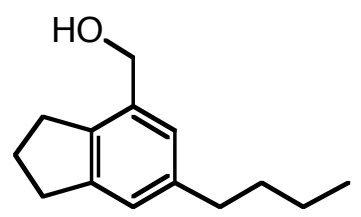

To a solution of $\mathbf{6 a}$ (100 mg, $0.32 \mathrm{mmol}, 1$ equiv) in THF (4 mL) was added TBAF (1 M solution in THF, $0.70 \mathrm{~mL}, 0.64 \mathrm{mmol}, 2$ equiv). The mixture was refluxed until the completion of the reaction by TLC. The solution was then diluted with $\mathrm{Et}_{2} \mathrm{O}$, washed with a saturated solution of $\mathrm{NH}_{4} \mathrm{Cl}$, brine, dried over $\mathrm{MgSO}_{4}$, filtered and concentrated in vacuo to give $\mathbf{1 4}(64 \mathrm{mg}, \mathbf{1 0 0 \%})$ as a white solid.

m.p. $49-52^{\circ} \mathrm{C}$

${ }^{1} \mathrm{H}$ NMR (400 MHz, $\mathrm{CDCl}_{3}$ ) $\square 7.06$ (br s, 2H), 4.67 (s, 2H), 2.92 (q, J = $\left.7.6 \mathrm{~Hz}, 4 \mathrm{H}\right), 2.63$ $(\mathrm{t}, J=7.7 \mathrm{~Hz}, 2 \mathrm{H}), 2.12(\mathrm{qt}, J=7.5 \mathrm{~Hz}, 2 \mathrm{H}), 1.62(\mathrm{~m}, 2 \mathrm{H}), 1.41(\mathrm{~m}, 2 \mathrm{H}), 0.97(\mathrm{t}, J=7.4$ $\mathrm{Hz}, 3 \mathrm{H})$.

${ }^{13} \mathrm{C}$ NMR (100 MHz, $\left.\mathrm{CDCl}_{3}\right) \square 144.9,141.6,139.5,136.1,125.1,123.9,63.8,35.7,34.2$, $32.8,30.5,25.3,22.6,14.1$.

IR (neat) 3360, 2927, 1457, 1057, $860 \mathrm{~cm}^{-1}$.

HRMS calcd for $\mathrm{C}_{14} \mathrm{H}_{20} \mathrm{O}\left([\mathrm{M}-\mathrm{OH}]^{+}\right):$187.1409. Found 187.1409.

[6-Butyl-5-(diisopropyl-methyl-silanyl)-indan-4-yl]-methanol (15):

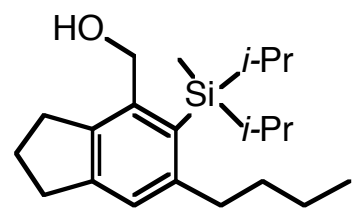

To a cooled $\left(0^{\circ} \mathrm{C}\right)$ solution of $\mathbf{6 a}(100 \mathrm{mg}, 0.32 \mathrm{mmol}, 1$ equiv) in $6 \mathrm{~mL}$ of $\mathrm{THF}$ was added MeLi. (1.6 $\mathrm{M}$ in $\mathrm{Et}_{2} \mathrm{O}, 0.4 \mathrm{~mL}, 0.63 \mathrm{mmol}, 2$ equiv). The mixture was warmed to room temperature. After the reaction was completed by TLC, the solution was diluted with $\mathrm{Et}_{2} \mathrm{O}$, washed successively with a saturated solution of $\mathrm{NH}_{4} \mathrm{Cl}$ and brine, dried over $\mathrm{MgSO}_{4}$, filtered, concentrated in vacuo to furnish $15(104 \mathrm{mg}, \mathbf{1 0 0 \%})$ as a yellow oil.

${ }^{1} \mathrm{H}$ NMR (400 MHz, CDCl$\left.)_{3}\right) \square 7.11(\mathrm{~s}, 1 \mathrm{H}), 4.73$ (s, 2H), $3.04(\mathrm{t}, J=7.4 \mathrm{~Hz}, 2 \mathrm{H}), 2.94$ (t, $J=7.5 \mathrm{~Hz}, 2 \mathrm{H}), 2.72(\mathrm{t}, J=8.0 \mathrm{~Hz}, 2 \mathrm{H}), 2.10(\mathrm{~m}, 2 \mathrm{H}), 1.58-1.34(\mathrm{~m}, 4 \mathrm{H}), 1.13$ (br s, $7 \mathrm{H}), 1.99$ (t, $J=7.3 \mathrm{~Hz}, 3 \mathrm{H}), 0.93($ br s, $7 \mathrm{H}), 0.49(\mathrm{~s}, 3 \mathrm{H})$.

${ }^{13} \mathrm{C}$ NMR $\left(100 \mathrm{MHz}, \mathrm{CDCl}_{3}\right) \square 148.8,145.7,142.2,142.1,132.7,125.8,63.4,36.7,35.9$, 33.0, 31.7, 24.7, 23.2, 19.7 (2C), 19.1 (2C), 15.4 (2C), 14.3, -6.4.

IR (neat) $3316,2927,2861,1562,1013,782,710,634 \mathrm{~cm}^{-1}$.

Anal calcd for $\mathrm{C}_{21} \mathrm{H}_{36} \mathrm{OSi}$ :C, 75.84; H, 10.91. Found: C, 75.68; H, 11.06. 


\section{(4-Hydroxymethyl-phenyl)-methanol (16):}<smiles>OCc1ccc(CO)cc1</smiles>

To a solution of 8 (100 mg, $0.28 \mathrm{mmol}, 1$ equiv) in $4 \mathrm{~mL}$ of THF were added $1.4 \mathrm{~mL}$ of TBAF ( $1 \mathrm{M}$ solution in THF, $1.38 \mathrm{mmol}, 5$ equiv). The mixture was refluxed until the completion of the reaction by TLC. The solution was then diluted with $\mathrm{Et}_{2} \mathrm{O}$, washed with a saturated solution of $\mathrm{NH}_{4} \mathrm{Cl}$, brine, dried over $\mathrm{MgSO}_{4}$, filtered and concentrated in vacuo to afford $16(23 \mathrm{mg}, \mathbf{6 0 \%})$ as a white solid. ${ }^{2}$

\section{(6-Butyl-5-hydroxymethyl-biphenyl-2-yl)-methanol (17):}

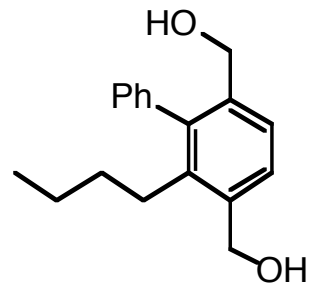

The procedure is the same as described above for 16. Diol 17 is obtained in $62 \%$ yield. ${ }^{1} \mathrm{H}$ NMR $\left(400 \mathrm{MHz}, \mathrm{CDCl}_{3}\right) \square 7.29(\mathrm{~m}, 5 \mathrm{H}), 7.06(\mathrm{~d}, J=6.8 \mathrm{~Hz}, 2 \mathrm{H}), 4.64(\mathrm{~s}, 2 \mathrm{H}), 4.17$ $(\mathrm{s}, 2 \mathrm{H}), 2.28(\mathrm{~m}, 2 \mathrm{H}), 1.22(\mathrm{~m}, 2 \mathrm{H}), 1.02$ (sextuplet, $J=7.4 \mathrm{~Hz}, 2 \mathrm{H}), 0.59$ (t, $J=7.4 \mathrm{~Hz}$, $3 \mathrm{H})$.

${ }^{13} \mathrm{C}$ NMR (100 MHz, $\left.\mathrm{CDCl}_{3}\right) \square 140.9,139.2,139.1,138.5,137.9,129.3$ (2C), 128.3 (2C), $127.5,127.1,125.0,63.5,63.0,33.2,29.1,22.9,13.5$.

IR (neat) 3281, 2927, 1460, 1047, 996, $702 \mathrm{~cm}^{-1}$.

\section{(6-Butyl-4-hydroxymethyl-biphenyl-2-yl)-methanol (18):}

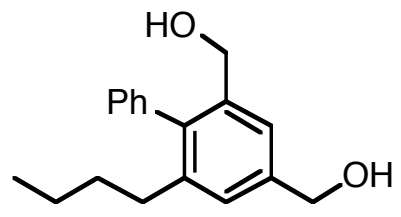

The procedure is the same as described above for 16. Diol 18 is obtained in $\mathbf{7 6 \%}$ yield. m.p. $66-68^{\circ} \mathrm{C}$

${ }^{1} \mathrm{H}$ NMR (400 MHz, CDCl $) \square 7.43(\mathrm{~m}, 4 \mathrm{H}), 7.24(\mathrm{~s}, 1 \mathrm{H}), 7.17(\mathrm{~m}, 2 \mathrm{H}), 4.71(\mathrm{~s}, 2 \mathrm{H})$, $4.34(\mathrm{~s}, 2 \mathrm{H}), 2.35(\mathrm{~m}, 4 \mathrm{H}), 2.35(\mathrm{~m}, 2 \mathrm{H}), 1.40(\mathrm{~m}, 2 \mathrm{H}), 1.18(\mathrm{~m}, 2 \mathrm{H}), 0.76(\mathrm{t}, J=7.3 \mathrm{~Hz}$, $3 \mathrm{H})$.

\footnotetext{
${ }^{2}$ NMR spectra, melting point and IR are identical to those described, see: Horner, L.; Weissbach, R. Justus Liebigs Ann. Chem. 1972, 757, 69-74.
} 
${ }^{13} \mathrm{C}$ NMR $\left(100 \mathrm{MHz}, \mathrm{CDCl}_{3}\right) \square 141.6,140.2,139.5,139.2,138.9,129.4(2 \mathrm{C}), 128.3(2 \mathrm{C}), 127.1,126.8$, $123.5,65.2,63.5,33.5,33.0,22.5,13.8$.

IR (neat) $3238,2955,2920,2853,1459,1052,700 \mathrm{~cm}^{-1}$.

HRMS calcd for $\mathrm{C}_{19} \mathrm{H}_{22} \mathrm{O}_{2}\left([\mathrm{M}+\mathrm{Na}]^{+}\right)$: 293.1517. Found: 293.1542 . 